\title{
Effects of Polarity for Monophasic and Biphasic Shocks on Defibrillation Efficacy with an Endocardial System
}

\author{
MASAHIRO USUI, GREGORY P. WALCOTT, S. ADAM STRICKBERGER, * \\ DENNIS L. ROLLINS, WILLIAM M. SMITH, and RAYMOND E. IDEKER \\ From the Departments of Medicine and Pathology, Duke University Medical Center, and the \\ Engineering Research Center for Emerging Cardiovascular Technologies of the School of \\ Engineering, Duke University, Durham, North Carolina; and the *Department of Cardiology, \\ University of Michigan Medical Center, Ann Arbor, Michigan
}

\begin{abstract}
USUI, M., ET AL.: Effects of Polarity for Monophasic and Biphasic Shocks on Defibrillation Efficacy with an Endocardial System. Electrode polarity has been reported to be one of the factors that affect defibrillation efficacy. We studied the influence of polarity on defibrillation efficacy when monophasic and biphasic waveforms were used with an endocardial lead system. In six anesthetized pigs, defibrillation catheters were placed in the right ventricular (RV) apex and at the junction of the superior vena cava (SVC) and right atrium. Monophasic shocks were $6 \mathrm{~ms}$ in duration, while for biphasic shocks the first phase was 6 $\mathrm{ms}$ and the second was $4 \mathrm{~ms}$ in duration. Four electrode configurations were tested: R:S, $M$ (the RV electrode, cathode; the SVC electrode, anode, with a monophasic shock); S:R, M; R:S, B (the RV electrode, first phase cathode; the SVC electrode, first phase anode, with a biphasic shock); $S: R, B$. Defibrillation probability of success curves were determined using an up/down protocol requiring 15 shocks for each configuration. For monophasic shocks, total delivered energy at the $50 \%$ probability of success point was significantly lower when the RV electrode was an anode than when it was a cathode ( $R: S, M: 24.4 \pm 7.4$ $J$ [mean $\pm S D$ ] vs $S: R, M: 16.4 \pm 5.5 \mathrm{~J} ; P<0.05$ ). For biphasic shocks, total energy was not affected by polarity reversal of the electrodes ( $R: S, B: 8.7 \pm 1.4 \mathrm{~J}$ vS $S: R, B: 8.4 \pm 2.5 \mathrm{~J} ; P=N S$ ). The endocardial electrode configuration with the $R V$ electrode as an anode requires less energy for defibrillation with $a$ monophasic but not a biphasic waveform. (PACE 1996; 19:65-71)
\end{abstract}

\section{transvenous defibrillation, polarity reversal, monophasic waveform, biphasic waveform}

\section{Introduction}

At implantation of implantable cardioverter defibrillators (ICDs), several factors influence defibrillation effectiveness including the $\operatorname{size}^{1}$ and location $^{2}$ of the defibrillation electrodes, the waveform $^{3,4}$ and the sequence of delivery ${ }^{5}$ of the defibrillation pulse. In patch electrode systems,

Supported in part by the National Institutes of Health research grants HL-42760, HL-44066, by Cardiac Pacemakers, Inc. (St. Paul, MN), and by the Japan Foundation of Aging and Health (Tokyo, Japan).

Address for reprints: Raymond E. Ideker, M.D., Ph.D., Cardiac Rhythm Management Laboratory, Volker Hall G78A, 1670 University Blvd., Birmingham, AL 35294-0019. Fax: (205) 9754720.

Received September 6, 1994; revision December 5, 1994; accepted December 22, 1994. electrode polarity is another factor reported to affect defibrillation efficacy. ${ }^{6-8}$ Optimal polarity has been reported to vary from patient to patient so it is recommended that both polarities be assessed at the time of ICD implantation. ${ }^{7-9}$ For external defibrillation, electrode polarity does not appear to affect cardiac resuscitation. ${ }^{10}$ Recent reports suggest that the defibrillation threshold (DFT) is lower with the right ventricular (RV) electrode as an anode than with the RV electrode as a cathode in a catheter-to-subcutaneous patch system in dogs with monophasic and biphasic shocks ${ }^{11}$ and in a totally catheter-based system in humans with monophasic shocks. ${ }^{12}$ The purpose of this study was to determine probability of success curves for defibrillation in pigs to evaluate whether the electrode polarity of monophasic and biphasic shocks affects defibrillation efficacy with an endocardial 
lead system having electrodes in the superior vena cava (SVC) and RV.

\section{Methods}

This study was approved by the Institutional Animal Care and Use Committee at Duke University. It conformed to the guidelines of the American Heart Association on Research Animal Use adopted November 11, 1984.

\section{Animal Preparation}

Six, 23- to $27-\mathrm{kg}$ pigs were preanesthetized with $74 \mathrm{mg} / \mathrm{kg}$ ketamine and $0.74 \mathrm{mg} / \mathrm{kg}$ acepromazine, given intramuscularly. Subsequently, anesthesia was maintained with sodium pentobarbital by a $10 \mathrm{mg} / \mathrm{kg}$ initial intravenous dose followed by a continuous intravenous infusion of $0.05 \mathrm{mg} /$ $\mathrm{kg}$ per minute. Succinylcholine was initially given at an intramuscular dose of $1 \mathrm{mg} / \mathrm{kg}$ and later at $0.25-0.5 \mathrm{mg} / \mathrm{kg}$ no more than once per hour to decrease muscle contraction induced by defibrillation shock. Morphine sulfate was initially given at an intramuscular dose of $0.4 \mathrm{mg} / \mathrm{kg}$ and later at 0.2 $\mathrm{mg} / \mathrm{kg}$ once per 2 hours to maintain analgesia. The pigs were intubated with a cuffed endocardial tube and ventilated with room air and oxygen through a respirator (Harvard Apparatus, Inc., South Natick, MA, USA). Arterial blood pressure was monitored with a catheter inserted in the femoral artery and connected to a Statham transducer (Gould, Inc., Valley View, OH, USA). Blood pressure and a lead II electrocardiogram were continuously displayed on a monitor (VSM, Physio-Control Corp., Redmond, WA, USA). Rectal temperature was monitored continuously and maintained within normal limits with an electric blanket. Normal saline was continuously infused through a catheter placed in the right internal jugular vein. Blood samples were taken every 60 minutes to determine the $\mathrm{pH}, \mathrm{P}_{\mathrm{O} 2}$, $\mathrm{P}_{\mathrm{CO} 2}$, base excess, $\mathrm{CO}_{2}, \mathrm{HCO}_{3}{ }^{-}$contents, and calcium, potassium, and sodium concentrations. Normal metabolic status was maintained throughout the study by administrating electrolytes and changing the oxygen concentration of inspired air.

Two 3.4-cm long catheter platinum-coated titanium electrodes, $3.9 \mathrm{~cm}^{2}$ in area (Cardiac Pacemakers, Inc., St. Paul, MN, USA) were used. One was inserted from the left external jugular vein and the other was inserted from the right external jugular vein. These two electrodes were randomly exchanged for each experiment. The electrodes were advanced to the proper position under fluoroscopic guidance. The SVC electrode was positioned at the right atrial/SVC junction. The RV electrode was advanced as far as possible into the $\mathrm{RV}$ apex. Each catheter was secured with a ligature at the venotomy site to stabilize its position.

\section{Fibrillation and Defibrillation Procedures}

Ventricular fibrillation was induced with 60$\mathrm{Hz}$ alternating current delivered through the two defibrillating catheters. Fibrillation was allowed to continue 10 seconds before a defibrillation test shock was given. Defibrillation testing was performed during expiration with the respirator temporarily disconnected from the intubation tube. If a test shock failed, a rescue shock of higher defibrillation energy was given immediately through the same catheter system. A minimum of 4 minutes was allowed to elapse before another shock was tested.

Monophasic or biphasic, fixed duration, truncated exponential shocks were generated by a 150$\mu \mathrm{F}$ defibrillator (Ventritex HVS-02, Sunnyvale, CA, USA). Monophasic shocks were $6 \mathrm{~ms}$ in duration, while for biphasic shocks the first phase was $6 \mathrm{~ms}$ and the second was $4 \mathrm{~ms}$ in duration (Fig. 1). For biphasic shocks, leading edge voltage of phase two (V2L) equaled the trailing edge voltage of phase one (V1T) (Fig. 1), and the overall tilt varied with system impedance.

Four electrode configurations were tested (Fig. 2):

1. The RV electrode (R), cathode $(-)$ to the SVC electrode (S), anode $(+)$, monophasic waveform (R:S, M); $\mathrm{R}, \mathrm{M})$;

2. $\mathrm{S}(-)$ to $\mathrm{R}(+)$, monophasic waveform (S:

3. R first phase (-) to $S$, first phase $(+)$, biphasic waveform (R:S, B);

4. $\mathrm{S}(-)$ to $\mathrm{R}(+)$, biphasic waveform each experiment.

The sequence of order for testing electrode configurations was randomized to account for variation during the course of the experiment. Each trial consisted of four shocks of these con- 

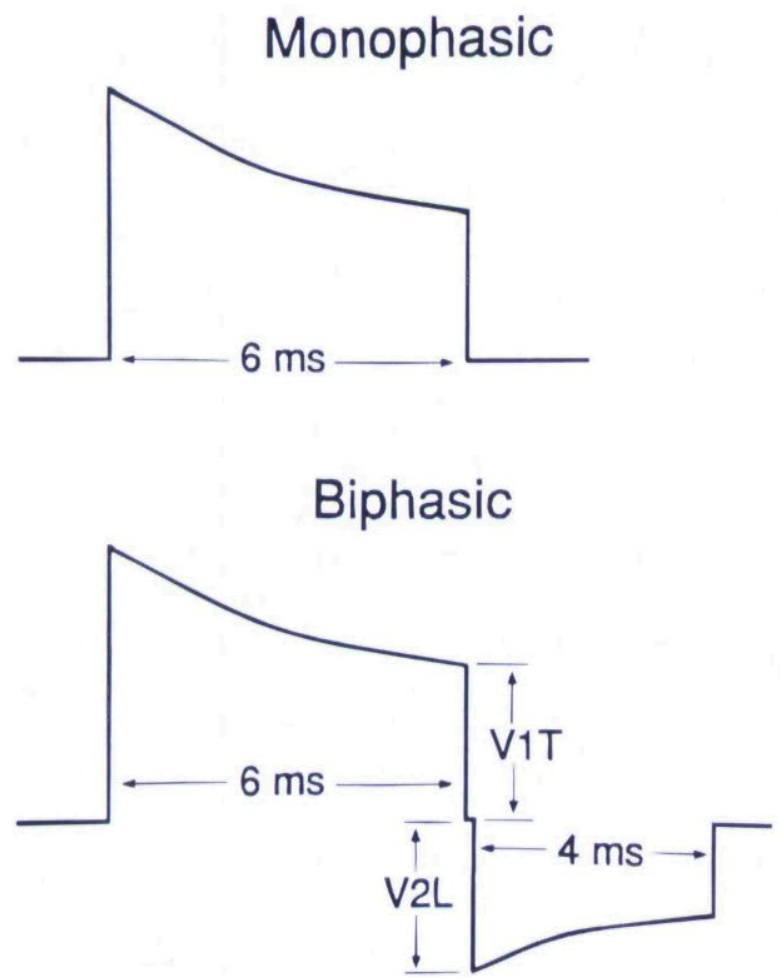

Figure 1. Waveforms for defibrillation shocks. The monophasic waveform was $6 \mathrm{~ms}$ and the biphasic waveform was 6/4 ms in duration. For the biphasic waveform, leading edge voltage of phase two (V2L) equaled the trailing edge voltage of phase one (V1T). Time between phases of the biphasic shock was $0.2 \mathrm{~ms}$.

figurations. The four shocks in each trial were delivered in random order.

A probability of success method that used an up/down protocol was used to determine defibrillation efficacy. ${ }^{13-15}$ An up/down protocol requiring 15 shocks was used to determine each defibrillation probability of success curve. Counting of the 15 shocks was not started until the first reversal in response. The starting voltage was $600 \mathrm{~V}$ for monophasic shocks and $400 \mathrm{~V}$ for biphasic shocks. Voltage steps of $40 \mathrm{~V}$ were used initially. Every time a shock succeeded, the next test shock was decreased by one step size. Conversely, every time a shock failed, the next shock was increased by one step size. Step size was reduced to $20 \mathrm{~V}$ following the first reversal of outcome from success to failure or from failure to success.

At the conclusion of the study, the anesthetized animal was terminally fibrillated. The chest was opened, and the positions of the catheters were checked to verify their locations. The catheters were removed, and the heart was excised and weighed.

\section{Data Analysis}

The actual current and voltage waveforms delivered to the electrodes were obtained by isolating and recording the voltage across a 0.25 -ohm resistor in series with the electrodes and a 200:1, 100 M-ohm resistor divider in parallel with the electrodes. These waveforms were digitized at a frequency of $20 \mathrm{kHz}$ and recorded by a waveform analyzer (model 6100, Data Precision, Inc., Danvers, MA, USA). Signal analysis software within the analyzer was used to calculate the impedance and energy from the current and voltage measurements. The output of the waveform analyzer was stored in a computerized datafile (Sun Microsystems, Inc., Mountain View, CA, USA).

For monophasic shocks, voltage was expressed as leading edge voltage, current as the average current, and energy as the total delivered energy. For biphasic shocks, voltage was expressed as leading edge voltage of phase one, current as the average current of phase one, and energy the
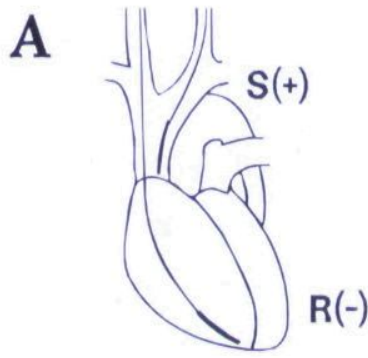

R:S, M
B

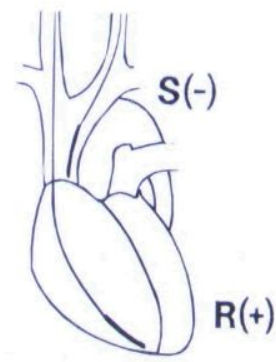

$\mathrm{S}: \mathrm{R}, \mathrm{M}$
Figure 2. Electrode configurations. A superior vena caval (SVC) electrode (S) was positioned at the right atrial/SVC junction, and a right ventricular (RV) electrode $(R)$ was placed in the RV apex. Four configurations were tested: $R$ cathode (-) to $S$ anode $(+)$, monophasic waveform (R:S, M) (panel A); $S(-)$ to $R(+)$, monophasic waveform (S:R, $M$ ) (panel B); $R$ first phase (-) to $S$ first phase (-) to $R$ first phase $(+)$, biphasic waveform $(R: S, B)$ (panel A); $S$ first phase $(+)$, biphasic waveform (S:R, B) (panel B). 
sum of the delivered energy of both phases of the test shock.

For each animal, sigmoid defibrillation probability curves were generated for each measured parameter using probit analysis. ${ }^{16}$ The mean values \pm SD for leading edge voltage, total delivered energy, and average current were determined at the $50 \%$ and $80 \%$ probability of success points for each configuration. Impedance was calculated by averaging the data from the 15 shocks for each configuration in each animal. The mean values \pm SD of impedance were determined for each configuration. Repeated measures analysis of variance with the Student-Newman-Keul's test ${ }^{16}$ was used to compare these variables. Significance was defined as $\mathrm{P}<0.05$.

\section{Results}

The mean heart weight \pm SD was $138 \pm 22$ g for six pigs. Voltage, energy, and current at the $50 \%$ and $80 \%$ success points and the impedance for each configuration are summarized in Table I.

For monophasic shocks, the polarity reversal of the RV electrode from cathode to anode caused a significant decrease in voltage, energy, and current. At the $50 \%$ success point, voltage and current were decreased by $18 \%$, and energy was decreased by $33 \%$. Polarity reversal did not affect the interelectrode impedance.

For biphasic shocks, which were $4 \mathrm{~ms}$ longer in total duration than monophasic shocks, voltage, energy, and current all were significantly lower than for monophasic shocks. However, polarity re- versal of the RV electrode from first phase cathode to first phase anode did not significantly affect defibrillation efficacy. Although interelectrode impedance was slightly higher than it was for monophasic shocks probably because the shock voltage was lower, ${ }^{17}$ polarity reversal did not affect the interelectrode impedance, either.

\section{Discussion}

This study compared the influence of polarity on defibrillation efficacy for monophasic and biphasic shocks with an endocardial electrode configuration. For monophasic shocks, defibrillation energy requirements were lower when the RV electrode was the anode than when it was the cathode. For biphasic shocks, defibrillation energy requirements were not different whether the RV electrode was a first anode or a cathode.

\section{Influence of Polarity Reversal for Monophasic Shocks}

Defibrillation energy requirements were lower with the RV electrode as an anode for monophasic shocks. We used two electrodes of the same type as the SVC and RV electrodes. Thus, the results cannot be explained by differences in the size, surface area, or material of the electrodes since they were the same for both electrodes. Changes in impedance cannot explain these results either, because interelectrode impedances were not affected by polarity reversal. Therefore, different responses of the myocardium to the two

Table I.

Effects of Polarity on Defibrillation Probability of Success and Impedance

\begin{tabular}{|c|c|c|c|c|c|c|c|}
\hline \multirow[b]{2}{*}{ Configuration } & \multicolumn{3}{|c|}{$50 \%$ Success } & \multicolumn{3}{|c|}{$80 \%$ Success } & \multirow{2}{*}{$\begin{array}{c}\text { Impedance } \\
\text { Ohms }\end{array}$} \\
\hline & Volts & Joules & Amps & Volts & Joules & Amps & \\
\hline $\mathrm{R}: \mathrm{S}, \mathrm{M}$ & $664 \pm 92$ & $24.4 \pm 7.4$ & $8.26 \pm 1.28$ & $720 \pm 91$ & $27.6 \pm 7.6$ & $8.66 \pm 1.21$ & $61.7 \pm 3.4$ \\
\hline$S: R, M$ & $547 \pm 88^{\star}$ & $16.4 \pm 5.5^{\star}$ & $6.78 \pm 0.99^{\star}$ & $602 \pm 75^{*}$ & $19.4 \pm 4.9^{\star}$ & $7.41 \pm 1.22^{*}$ & $61.9 \pm 3.2$ \\
\hline $\mathrm{R}: \mathrm{S}, \mathrm{B}$ & $362 \pm 19^{\star \star}$ & $8.7 \pm 1.4^{\star \star}$ & $4.42 \pm 0.51^{\star \star}$ & $395 \pm 58^{\star \star}$ & $10.1 \pm 3.0^{\star \star}$ & $4.64 \pm 0.67^{\star \star}$ & $63.0 \pm 3.2^{\star \star}$ \\
\hline$S: R, B$ & $357 \pm 48^{\star \star}$ & $8.4 \pm 2.5^{\star \star}$ & $4.30 \pm 0.62^{\star \star}$ & $406 \pm 74^{\star \star}$ & $10.6 \pm 4.0^{* *}$ & $4.75 \pm 0.90^{\star *}$ & $62.7 \pm 3.0^{\star \star}$ \\
\hline
\end{tabular}

Configurations are in Figure 2. Values are mean \pm SD.

${ }^{*} \mathrm{P}<0.05$ when compared to R:S, M.

${ }^{* \star} \mathrm{P}<0.05$ when comparing $\mathrm{S}: \mathrm{R}, \mathrm{M}$ versus $\mathrm{R}: \mathrm{S}, \mathrm{B}$, and $\mathrm{S}: \mathrm{R}, \mathrm{M}$ versus $\mathrm{S}: \mathrm{R}, \mathrm{B}$. 
shock polarities must be responsible for the different defibrillation efficacies.

Schuder et al. ${ }^{6}$ found that the percentage of successful defibrillation was slightly higher when the upper RV patch was used as an anode than when the left ventricular (LV) apical patch was used as an anode in dogs. In contrast, Bardy et al. ${ }^{7}$ reported that the DFT in terms of delivered energy was $23 \%$ lower when the posterolateral LV patch was positive than when the anterior RV patch was positive during clinical implantation of ICDs. The different results between these studies might be explained by the different positions of the LV patch. When the LV patch is placed on the apex, defibrillation efficacy would be greater with the patch as a cathode. When the LV patch is placed on the posterolateral wall, defibrillation efficacy would be greater with the patch as an anode. Thakur et al. ${ }^{11}$ found that defibrillation energy requirements were lower when the RV catheter electrode was an anode than when the left chest wall subcutaneous patch was an anode in dogs. Schuder et al. ${ }^{6}$ found that for transthoracic defibrillation in calves the percentage of successful defibrillation was higher when the electrode over the apex was a cathode. However, Weaver et al. ${ }^{10}$ reported that for external defibrillation in humans polarity did not affect the outcomes of cardiac resuscitation. The results of these studies and our study suggest that defibrillation energy requirements may be lower when the electrode attached to the RV is used as an anode or when the left thoracic electrode over the apex is used as a cathode, but that in clinical practice other factors such as longer fibrillation time or the existence of myocardial infarction may minimize the effect of polarity on defibrillation efficacy.

The experimental results of this study support the findings of one family of mathematical models of the relationship between shock fields and the transmembrane potential called bidomain models. ${ }^{18,19}$ While predicting that a cathode depolarizes the transmembrane potential of cells immediately adjacent to the shocking electrode, these models also predict that, beginning 1 to $4 \mathrm{~mm}$ away from the cathode and extending for several centimeters or more, the transmembrane potential is hyperpolarized. Conversely, immediately adjacent to an anode the transmembrane potential is hyperpolarized, while tissue farther than 1-4 mm from the anode is depolarized. Recently, hyperpolarized regions near a cathode (virtual anodes) and depolarized regions near an anode (virtual cathodes) were observed experimentally in cardiac tissue. $^{20,21}$ Previous experimental results indicate that the ventricular region from which activation first appears after a failed defibrillation shock and in which the shock extracellular potential gradient is weak for the electrode configuration used in this study is the LV apex and LV lateral free wall. ${ }^{22,23}$ The bidomain models raise the possibility that this region is hyperpolarized when the RV electrode is a cathode and is depolarized when the RV electrode is an anode. If we assume similarly that it is easier to defibrillate depolarized tissue than hyperpolarized tissue with truncated exponential waveforms, defibrillation efficacy should be greater when the RV electrode is an anode, since this will cause the tissue in which the shock potential gradient is weak and in which earliest activation appears following failed defibrillation shocks to be depolarized. The results of this study confirm this prediction. This theory predicts that, if the SVC electrode is placed in the region of the innominate vein or in the coronary sinus with a totally catheter-based system, defibrillation efficacy should still be greater with the RV electrode as an anode because the anode should depolarize the LV apex. However, if an LV patch is placed on the apex or if a left thoracic electrode is placed over the apex, defibrillation efficacy should be greater with the patch or thoracic electrode as a cathode because the cathode should depolarize the LV apex underlying the electrode.

\section{Influence of Polarity Reversal for Biphasic Shocks}

As opposed to a monophasic shock, the biphasic waveform can depolarize all portions of the ventricles. Part of the tissue will be depolarized during the first phase of the shock; the remainder of the tissue, which is hyperpolarized during the first phase, will be depolarized by the second phase if the amount of current during the second phase of the shock is sufficiently large. Reversing the polarity of a biphasic shock simply interchanges which portions of the heart are depolarized by the first phase and by the second phase. Therefore, in contrast to monophasic shocks, re- 
versing shock polarity for biphasic shocks would not necessarily be expected to increase defibrillation efficacy if field requirements for defibrillation are lower for depolarized than hyperpolarized tissue. The finding in this study that biphasic shock efficacy was not altered by changing shock polarity supports this explanation.

Other findings, however, do not support this explanation. The biphasic waveform used in this study delivered much more current in the first phase than in the second phase. According to this explanation, reversing the sequence of the two phases should not affect defibrillation efficacy, since with either sequence the two phases of the biphasic waveform should depolarize all portions of the ventricles. It has been shown, however, that reversing the two phases of the biphasic waveform, so that the amount of current delivered during the first phase is much smaller than that delivered during the second phase, greatly decreases defibrillation efficacy. ${ }^{24,25}$

Thakur et al. ${ }^{11}$ reported that polarity reversal also affected defibrillation efficacy for biphasic shocks with the RV-subcutaneous patch configuration. Defibrillation energy requirements were lower when the RV catheter electrode was a first phase anode than when the left chest wall subcutaneous patch was a first phase anode. This discrepancy between their results and ours suggests that differences in electrode configurations may influence the effect of polarity on biphasic defibrillation.

\section{References}

1. Troup PJ, Chapman PD, Olinger GN, et al. The implanted defibrillator: Relation to defibrillating lead configuration and clinical variables to defibrillation threshold. J Am Coll Cardiol 1985; 6: 1315-1321.

2. Ideker RE, Wolf PD, Alferness CA, et al. Current concepts for selecting the location, size, and shape of defibrillation electrodes. PACE 1991; 14: 227-240.

3. Winkle RA, Mead RH. Ruder MA, et al. Improved low energy defibrillation efficacy in man with the use of a biphasic truncated exponential waveform. Am Heart J 1989; 117:122-127.

4. Bardy GH, Ivey TD, Allen MD, et al. A prospective randomized evaluation of biphasic versus monophasic waveform pulses on defibrillation efficacy in humans. J Am Coll Cardiol 1989; 14:728-733.

5. Bardy GH, Ivey TD, Johnson G, et al. Prospective

\section{Limitations}

Other waveforms of various duration were not tested. For biphasic shocks, a 6/4-ms waveform was chosen because waveforms with a shorter phase two than phase one duration are more effective for defibrillation. ${ }^{25,26}$ If biphasic waveforms with a longer or shorter second phase duration had been used, polarity reversal might have made a difference in defibrillation efficacy. Similarly, other defibrillation electrode configurations were not tested. Electrode location may also alter the effects of shock polarity on defibrillation.

\section{Conclusions}

The major findings from this study are: (1) for monophasic shocks, leading edge voltage, average current, and total delivered energy at $50 \%$ and $80 \%$ probability of success were significantly lower when the RV electrode was an anode than when it was a cathode with an SVC-RV endocardial system in pigs; and (2) for biphasic shocks, $50 \%$ and $80 \%$ probability of success values were not different whether the RV electrode was a first phase cathode or anode. Therefore, the configuration with the RV electrode as an anode should be tested first in clinical DFT testing when monophasic waveforms are used.

Acknowledgments: The authors would like to thank Ellen Dixon-Tulloch, Jenny Hagler, Letealia Oliver, and Kimberly Mulligan for their technical assistance.

evaluation of initially ineffective defibrillation pulses on subsequent defibrillation success during ventricular fibrillation in survivors of cardiac arrest. Am J Cardiol 1988; 62:718-722.

6. Schuder JC, Stoeckle H, McDaniel WC, et al. Is the effectiveness of cardiac ventricular defibrillation dependent upon polarity? Med Instrum 1987; 21 : 262-265.

7. Bardy GH, Ivey TD, Allen MD, et al. Evaluation of electrode polarity on defibrillation efficacy. Am J Cardiol 1989; 63:433-437.

8. O'Neill PG, Boahene KA, Lawrie GM, et al. The automatic implantable cardioverter-defibrillator: Effect of patch polarity on defibrillation threshold. J Am Coll Cardiol 1991; 17:707-711.

9. Troup P, Wetherbee JN, Chapman PD, et al. Does electrode polarity affect defibrillation efficacy? (abstract) PACE 1990; 13:528. 


\section{EFFECTS OF POLARITY ON DEFIBRILLATION}

10. Weaver WD, Martin JS, Wirkus MJ, et al. Influence of external defibrillator electrode polarity on cardiac resuscitation. PACE 1993; 16:285-291.

11. Thakur RK, Souza JJ, Chapman PD, et al. Electrode polarity is an important determinant of defibrillation efficacy using a nonthoracotomy system. PACE 1994; 17:919-923.

12. Strickberger SA, Hummel JD, Horwood LE, et al. Effect of shock polarity on ventricular defibrillation threshold using a transvenous lead system. J Am Coll Cardiol 1994; 24:1069-1072.

13. McDaniel WC, Schuder JC. An up-down algorithm for estimation of the cardiac ventricular defibrillation threshold. Med Instrum 1987; 21:170-176.

14. Davy JM, Fain ES, Dorian P, et al. The relationship between successful defibrillation and delivered energy in open-chest dogs: Reappraisal of the "defibrillation threshold" concept. Am Heart J 1987; 113:77-84.

15. Jones DL, Irish WD, Klein GJ, et al. Defibrillation efficacy: Comparison of defibrillation threshold versus dose-response curve determination. Circ Res 1991; 69:45-51.

16. SAS/STAT ${ }^{\circledR}$ User's Guide, Release 6.03 Edition. Cary, NC, SAS Institute, Inc., 1988.

17. Wharton JM, Wolf PD, Smith WM, et al. Cardiac potential and potential gradient fields generated by a single, combined, and sequential shocks during ventricular defibrillation. Circulation 1992; 85: 1510-1523.

18. Sepulveda NG, Roth BJ, Wikswo JP Jr. Current injection into a two-dimensional anisotropic bidomain. Biophys J 1989; 55:987-999.
19. Trayanova NA, Roth BJ, Malden LJ. The response of a spherical heart to a uniform electric field: A bidomain analysis of cardiac stimulation. IEEE Trans Biomed Eng 1993; 40:899-908.

20. Knisley SB, Hill BC, Ideker RE. Virtual electrode effects in myocardial fibers. Biophys J 1994; 66: 719-728.

21. Neunlist M, Tung L. Optical recordings of ventricular excitability of frog heart by an extracellular stimulating point electrode. PACE 1994; 17: 1641-1654.

22. Wolf PD, Walcott GP, Smith WM, et al. Epicardial mapping demonstrates a predictable arrhythmia following unsuccessful transvenous defibrillation near threshold. (abstract) J Am Coll Cardiol 1994; 23:421A.

23. Tang ASL, Wolf PD, Afework Y, et al. Three-dimensional potential gradient fields generated by intracardiac catheter and cutaneous patch electrodes. Circulation 1992; 85:1857-1864.

24. Dixon EG, Tang ASL, Wolf PD, et al. Improved defibrillation thresholds with large contoured epicardial electrodes and biphasic waveforms. Circulation 1987; 76:1176-1184.

25. Tang ASL, Yabe S, Wharton JM, et al. Ventricular defibrillation using biphasic waveforms: The importance of phasic duration. J Am Coll Cardiol 1989; 13:207-214.

26. Feeser SA, Tang ASL, Kavanagh KM, et al. Strength-duration and probability of success curves for defibrillation with biphasic waveforms. Circulation 1990; 82:2128-2141. 
This document is a scanned copy of a printed document. No warranty is given about the accuracy of the copy. Users should refer to the original published version of the material. 